\title{
Profesorado masculino en Educación Inicial de Ecuador: análisis del Archivo Maestro de Instituciones Educativas
}

\section{Male teachers in Initial Education of Ecuador: analysis of the Master File of Educational Institutions}

\author{
Johanna Mercedes Cabrera Vintimilla*1 y Josué Paul Cale Lituma ${ }^{1}$ \\ ${ }^{1}$ Universidad Nacional de Educación, Ecuador \\ *johanna.cabrera@unae.edu.ec
}

DOI: https://doi.org/10.26871/killkanasocial.v4i3.649

\begin{abstract}
Resumen
La investigación posee su génesis en los roles de género en la región occidental, específicamente en Ecuador. Este artículo tiene como objetivo demostrar cómo se sitúa al género masculino en el campo laboral de la Educación Inicial en Ecuador, en el que su desempeño es sinónimo de opresión y discriminación. En la metodología se toma como referencia a 100 Centros de Educación Inicial -CEI- del Archivo Maestro Instituciones Educativas -AMIE-, tanto del sector público como privado de la provincia de Azuay - Ecuador. En el que se evidencia la escasez de docentes de género masculino laborando en estos centros; la revisión de conceptos y resultados obtenidos, permite concluir que la insuficiencia de docentes masculinos en la educación de la primera infancia en Ecuador genera desigualdad laboral, social, opresora e incluso una óptica en el que el rol del hombre en educación queda estigmatizado por estereotipos sociales.
\end{abstract}

Palabras clave: educación inicial, roles de género, estereotipos, archivo maestro instituciones educativas.

\begin{abstract}
The research has its genesis in gender roles in the western region, specifically in Ecuador. This article aims to demonstrate how the male gender is situated in the field of Early Childhood Education in Ecuador, in which their performance is synonymous with oppression and discrimination. In the methodology, 100 Initial Education Centers -CEI-are taken from the Master File of Educational Institutions, both in the public and private sectors of the province of Azuay - Ecuador. In which the shortage of male teachers working in these centers is evident; the review of concepts and results obtained, allows us to conclude that the insufficiency of male teachers in early childhood education in Ecuador generates labor, social, oppressive inequality and even an optic in which the role of men in education is stigmatized by social stereotypes.
\end{abstract}

Keywords: initial education, gender roles, stereotypes, master file of educational institutions.

\section{Introducción}

En el Ecuador se considera a la Educación Inicial como una carrera para mujeres, puesto que aún se visibiliza gran cantidad de maestras laborando en el campo de la educación, especialmente con niños de la etapa inicial; pocos son los hombres que se arriesgan a romper con cánones creados por años en una sociedad, donde las carreras como ingenierías son concebidas como profesiones que desempeñan los hombres, dando relevancia al hombre en carreras técnicas, minimizando a la mujer; casi obligándola a seguir un perfil profesional que no exige fuerza física y que según la sociedad considera de menor esfuerzo. Estos estereotipos afectan también al hombre, puesto que él debe cumplir con una profesión impuesta primero por su familia y luego por los criterios que rigen una sociedad en la que no concibe a la educación inicial como una carrera para el hombre.

La escasa presencia de docentes varones en los centros de educación infantil resulta una población fácilmente contable. Sin embargo, en los últimos años en la educación se percibe cambios a pequeñas escalas del número de varones que laboran de manera directa con infantes entre 0 a 5 años. Se consideran que en países europeos no se supera el $5 \%$ de profesionales varones en educación infantil, mientras que Dinamarca y Noruega mejoran estas cifras (Vendrell, Dalmau, Gallego, y Baqués, 2015). La sociedad evoluciona en diferentes aspectos de la vida, en su mayoría en aspectos tecnológicos para la mejora de la calidad de vida, pero la evolución más difícil es la de transmutar los valores y el pensamiento en el ser humano, dejar de etiquetar a las personas, por su profesión. 
En países europeos esta evolución ha dado pasos gigantescos, lo que ha servido como apoyo y ejemplo para que otros sistemas apliquen esta idea, especialmente en la incursión del hombre en el campo de la educación inicial occidental. Por estos precedentes se ha considerado la relevancia del presente estudio; para demostrar la cantidad de docentes varones de Azuay - Ecuador, que laboran en Centros de Educación Inicial en comparación con docentes mujeres. Ventajosamente, en Ecuador se maneja el Archivo Maestro de Instituciones Educativas (AMIE), por lo que gracias a dicho documento nos hemos centrado en docentes varones en ejercicio dentro de Educación Inicial.

\subsection{Primera Infancia}

La UNESCO (2019) define a la primera infancia como el periodo que va desde el nacimiento hasta los ocho años de edad, mientras que la UNICEF (2017) afirma que la primera infancia es comprendida desde la concepción hasta el inicio de la educación escolar. Transcurso en el que los hechos que influyen de manera decisiva y son de suma importancia para el desarrollo cerebral.

Por ende, para el progreso intelectual, social y emocional de cada niño y niña es necesario garantizar un sistema educativo óptimo. A nivel internacional la proliferación de búsquedas hacia el acceso de la educación sin distinción es innumerable. Por ejemplo, el Objetivo de Desarrollo Sostenible (ODS) número cuatro de la Organización de Naciones Unidas -ONU- (2018) tiene como enfoque una Educación de Calidad, en el que la meta 4.2 Desarrollo de la primera infancia y educación preescolar universal es:

Para 2030, velar por que todas las niñas y todos los niños tengan acceso a servicios de atención y desarrollo en la primera infancia y a una enseñanza preescolar de calidad, a fin de que estén preparados para la enseñanza primaria. (UNESCO, 2019)

La primera infancia está instituida por un proceso, el cual, en psicología es conocida como psicología del desarrollo. Para Navarro y Pérez (2012) el desarrollo se refiere al tipo de cambio en el comportamiento desarrollado por su contexto, sociedad o cultura. Dicho cambio debe ocurrir en un recorrido espacio no solo individual, sino también contextual e histórico, en las dificultades que surgen en este proceso, de ahí que también se le conoce como "psicología evolutiva" (Navarro y Pérez, 2012).

Navarro y Pérez (2012) también añaden la importancia de la trayectoria histórica del sujeto, ya que esta va más allá de solo obtener una herencia -genética-. La herencia representa una forma para entender su continuidad y segundo, varios aspectos contextuales -que determinan la discontinuidad-: cultura, sociedad y tiempo -época en la que se desarrolla el niño o niña y la cultura que lo rodea-, ya que estos determinan la continuidad/discontinuidad en su ambiente e interacción con las personas.

Piaget describe al desarrollo como una "construcción progresiva" que se produce por la interacción del medio con el sujeto (Valhondo, 1995) y la asimilación de la acomodación entre la realidad y sus pensamientos (Gerrig y Zimbardo, 2005). Además, Piaget se interesaba en la forma de pensar de los niños según sus diferentes etapas de desarrollo (Gerrig y Zimbardo, 2005). Él plantea cuatro etapas del desarrollo infantil: etapa sensorio-motora - experiencias sensoriales físicas-, etapa preoperacional -se maneja el mundo de manera simbólica-, etapa de las operaciones concretas -capacidad limitada del razonamiento lógico- y etapa de las operaciones formales -capacidad de utilizar la lógica prepositiva y lógica abstracta- (Rice y Ortiz, 1997).

Por otra parte, para Vygotsky el desarrollo es concebido como el aumento gradual y cuantitativo de las proporciones físicas. Por ejemplo, el desarrollo del humano comprendido desde que es un homúnculo (Lenzi, Borzi, y Tau, 2010). De manera que estudia el recorrido vital continuo o evolutivo del ser humano, en el que también se destaca el proceso individual de construcción mental y el origen social de los procesos psicológicos superiores (Cuadernos UCAB, 1997).

Cornachione (2016) destaca que el desarrollo es una función histórica y cultural interactiva que está vinculada a la vida del ser humano. También que la teoría de desarrollo de Vygotsky determina la resolución de problemas, si se puede resolver de forma individual o si existe la necesidad de requerir ayuda/colaboración/guía de una persona capaz de resolver dicho problema. Por lo que el rol de la educación es proporcionar las herramientas, recursos, metodologías que sean necesarias para garantizar el progreso de la primera infancia de niños y niñas.

\subsection{Docentes y Educación Inicial en Ecuador}

El rol del docente en la Educación Inicial necesita algunas características específicas, según el Currículo de Educación Inicial (2014), donde se considera que la forma más importante para realizar un acercamiento con los niños es la forma de dialogar con ellos, utilizando un lenguaje sencillo para tender puentes entre lo que los niños saben y lo que el docente espera que aprendan.

Dentro de las características el docente debe ser auténtico, mostrar interés por conocer y entender qué piensan y qué desean los niños. Sus comentarios hacia el niño o grupo a cargo deben ser positivos y constructivos, que animen a continuar y a mejorar. El docente que trabaja con niños de 0-2 años, posee un rol de mediador, trabajan en función de las características particulares sus discentes, a más de estimular su desarrollo, deben atender de manera pertinente las necesidades de los infantes como: higiene, salud, alimentación, descanso y fortalecer la parte emocional de su edad, respetando el ritmo de aprendizaje de cada infante y su diversidad (Ministerio de Educación, 2014).

La educación en la actualidad debe estar libre de estereotipos que limitan el pensamiento del individuo, es decir que cada persona debe poseer un pensamiento crítico a la hora de seguir un plan de vida, dejar de encasillar a la Educación Inicial, como una carrera netamente para 
mujeres por un falso instinto materno que ha creado la sociedad, subyugando a la mujer para que se encargue de la labor con los niños; más bien se debe repensar en la importancia del hombre en la primera infancia, y creer en una educación como práctica de libertad. Freire (2014) en la pedagogía del oprimido asegura que:

Los métodos de opresión no pueden, contradictoriamente, servir a la liberación del oprimido. En esas sociedades, gobernadas por intereses de grupos, clases y naciones dominantes, "la educación como práctica de la libertad". No pedagogía para él, sino de él. Los caminos de la liberación son del mismo oprimido que se libera: él no es cosa que se rescata sino sujeto que se debe autoconfigurar responsablemente. La educación libertadora es incompatible con una pedagogía que, de manera consciente o mistificada, ha sido práctica de dominación. (p. 1)

Lo que indica que es responsabilidad netamente de la persona, que deja que la sociedad influya en sus decisiones de vida, especialmente en el campo laboral y de educación, formando falsos conceptos relacionados a la Educación Inicial, permitiendo que grupos encasillen a un género, dándole un rol determinado como en el caso de la presente investigación donde se observa que el rol de la mujer sigue primando en la educación de los primeros años del infante.

Existe una fuerte tendencia en la educación infantil, normalizada para la sociedad, pero de relevancia para las investigaciones sobre prácticas y cuidados diarios en guarderías, el de estigmatizar o delimitar el cuidado y educación como transposición de saberes encasillados como femeninos, adquiridos por las mujeres de manera cultural a través de la socialización primaria, la familia, el contexto, el ambiente y de sobre manera las experiencias procedentes del universo doméstico (Carvalho, 1999).

Dichos conceptos se siguen utilizando en la contemporaneidad y de a poco se están transformando a través de las nuevas masculinidades, que involucra al hombre como actor responsable, más no como un ser superior que debe realizar tareas consideradas como ayuda (Cappi y Salles, 2014).

\subsection{Presencia masculina en la primera infancia}

Cuando se piensa en la palabra escuela, generalmente se relaciona con espacios femeninos puesto que son lugares donde actúan e interactúan mayormente mujeres, especialmente cuando se relaciona con la temática de los primeros años escolares. En estos contextos las mujeres priman, ya que se idealiza la organización, la ocupación de estos espacios y la actividad escolar como un contexto donde aflora la función materna según los conceptos erróneos creados por la sociedad y su disyuntiva lógica.

Una investigación en la Universidad de Ciencias Empresariales y Sociales de Argentina afirma que, aunque hay hombres que se gradúan de la carrera de Pedagogía, es común que estos trabajen en otras áreas ajenas a la
Educación Inicial (Cappi y Salle, 2018). Además, Cappi y Salle (2018) mencionan que "los maestros al ingresar a las escuelas, son direccionados a funciones de apoyo o trabajo con grupos de mayor edad que demandan menos acciones con el cuidado corporal" (p.7). Se considera esta particularidad con el hecho de que las escuelas poseen un ambiente mayoritariamente femenino, y se relaciona la idea entre educación y maternidad (Manzini, 2017, citado en Cappi y Salle, 2018).

Esta concepción se ha concretizado en la tríada: mujermadre-maestra, que es evidente en la educación infantil por diversas concepciones sociales. Entonces, se relaciona la poca presencia masculina en la educación infantil en Brasil, con muchos debates sobre los factores que caracterizan este panorama, que fundamentan el rechazo a la actuación de los hombres en este nivel de enseñanza, puesto que es considerado "normal" el que un maestro trabaje en grados superiores que en la Educación Inicial.

La profesión, el proyecto de vida, los ideales de un individuo ya sea mujer u hombre y la docencia en la Educación Inicial se construyen diariamente, tanto de pensamientos de hombres y mujeres en las diferentes instituciones educativas. Es decir, no están solamente determinados por una 'estructura de género' que diseñaría la profesión a priori, en la cual centra a la mujer como actor principal para el trabajo con menores de cinco años, sino que están determinados por las ideologías preconcebidas socialmente.

\subsection{Imaginarios sociales}

Los imaginarios sociales son de interés significativo, puesto que la forma de entender cómo funciona la sociedad se basa de nuestros pensamientos, de cómo preconcebimos la estructura y función que desempeña cada persona. Es decir, cómo las personas imaginan su entorno social, lo que no se expresa en teoría, sino en a través de imágenes, historias y leyendas (Taylor, 2004). A más de esto podemos observar que en la cultura occidental existe un auge de tradiciones que determinan a la educación de la primera infancia correspondida de manera prioritaria a la mujer.

Sin embargo, este tipo de tradicionalismo ha sido forjado con leyendas, tradiciones, costumbres y sobre todo con la imagen de la mujer al frente de la Educación Inicial. Creando una doble moral, así como un imaginario encubridor de desigualdad social, y que el rol de hombres en el campo de la Educación Inicial queda subestimado y menospreciado. Szurmuk y McKee (2009) describen la interrelación de lo global-local para los imaginarios sociales, en los que:

(...) no supone un mundo sin fronteras donde desaparecen las particularidades locales, sino una nueva dimensión de los imaginarios culturales donde se disuelve el nexo conceptual entre cultura y territorio particular. Las interconexiones que perfilan un imaginario cultural global no sustituyen ni desplazan las culturas locales, pero las afectan al alterar las fronteras tradicionales de discursos y 
prácticas. El proceso que informa la configuración de ese nuevo imaginario se ha conceptualizado como hibridación o glocalización, entre otras formas. (p. 166)

Por tanto, la era de la globalización, interconexión e internet deberían facilitar la reacción al ver a hombres trabajar en el área de la Educación Inicial. El catalizador social principal para ampliar la perspectiva de los imaginarios debe ser, sin duda alguna, la educación de calidad y calidez, basadas en prácticas que irrumpan la continuidad de la construcción de imaginarios sociales encubridores de desigualdades, y, por ende, deconstruir una sociedad que, hasta el momento, tiene pilares de doble moral.

Por otra parte, las consideraciones de Scott (1995) indican que a partir de la noción de género es posible comprender una determinada sociedad tomando como base la construcción de los significados de masculino y femenino y la forma como ellos se reflejan en la organización de la vida social. Es decir, se forma una estructura social en la que la desigualdad tendrá como antecedente la falsa percepción de roles que desempeña cada persona en la sociedad.

\subsection{Masculinidades}

Los imaginarios sociales por los que atraviesa la educación de la primera infancia aún requieren un cambio abismal, la modernidad exige nuevos parámetros al momento de ingresar a una carrera considerada por la sociedad "para mujeres", por lo que las medidas que deben ser tomadas deben ser precisas y objetivas en su propósito para desviar la vista del imaginario social tradicional.

Se considera a varones como sujetos genéricos, sus relaciones como hombres es una construcción social y no un hecho de la naturaleza, por lo que la masculinidad, hombría no llegan y no pueden tener un significado estático o definido, pues el significado varía según el contexto, época o momento histórico (Nuñez, 2007), que determinan un significado contemporáneo que se encontrará en cambio constante.

La importancia de la construcción del significado de hombre y sus acciones ha sido motivo de estudios de género, en los que siempre interviene la sociedad, cultura y psicología, que subyacen el objetivo y el resultado de tal búsqueda. Núñez (2016) describe este proceso como

(...) un drama complejo que no puede reducirse, sino a riesgo de simplificarlo, en el concepto . estudio de las masculinidades", así se diga en plural y no en singular. $\mathrm{Y}$ es que no todos los varones son "masculinos. ${ }^{\circ}$ no lo somos de la misma manera; todos, sin embargo, somos afectados por ese dispositivo de poder de género. Desde esta perspectiva sociocultural, posestructuralista, actualmente el paradigma dominante en los estudios de género de los hombres y la masculinidad, "hombrez "masculinidad", deben ser tratados como términos en disputa; términos vacíos en sí mismos, pero rebosantes para el género. (p. 20)

Sin embargo, el rol masculino cada vez se hace más visible en la atención hacia discentes, la educación y el desarrollo infantil integral; no como rol paterno, sino más bien como el de educador, rompiendo así estereotipos sociales y de a poco involucrarse en la Educación Inicial y la estimulación temprana.

Por otro lado, las nuevas masculinidades han abierto un universo donde los hombres rompen etiquetas de estructuras sociales, concebidas desde hace muchos en el campo de la Educación Inicial. Connell (2003, citado en Ayala, 2007) menciona que la masculinidad es un proceso de relación entre estructuras sociales, prácticas y experiencias de cada etapa de la vida -en determinado contexto-. El autor hace énfasis en tres esferas: 1) esfera productiva -designación de tareas por género-, 2) esfera de poder -subordinación de la mujer ante el hombre- y 3) esfera de catexis o cathesis - se relaciona con la vida sexual activa socialmente hablando-.

La concepción de las masculinidades proviene de una óptica social hacia determinadas acciones y situaciones que no son comunes en un contexto, causando un grupo minoritario susceptible a discriminación, como lo es con hombres que ejercen labor en educación de la primera infancia.

\section{Metodología}

La investigación tuvo como propósito el evidenciar, analizar y comparar el número de docentes masculinos que trabajan en Centros de Educación Inicial con relación al número de mujeres que trabajan en el mismo espacio, de la provincia del Azuay - Ecuador, para lograr esto se trabajó con el enfoque cuantitativo. Herández, Fernández, y Batista (2014, citado en; Canto y Silva, 2012) definen a la investigación cuantitativa como "la recolección de datos para probar hipótesis con base a una medición numérica y el análisis estadístico, para establecer patrones de comportamiento y probar teorías" (p. 4); una de las características de este enfoque es la rigurosidad en el proceso de investigación, la información recolectada de manera estructurada y sistemática.

Otra característica esencial es la utilización de la lógica deductiva para identificar leyes causales o universales en una realidad externa al individuo o individuos motivo de estudio (Canto y Silva, 2012). En este sentido, la información que recolectamos será manifestada y expuesta en porcentajes y tablas, de manera que se pueda interpretar y realizar su análisis.

El método de la investigación corresponde al no experimental. Dicho método lo justificamos ya que es una búsqueda empírica y sistemática, en la que como investigadores no poseemos el control de las variables, pero sí podemos obtener inferencias respecto a las variables, tanto dependiente como independiente (Kerlinger y Howard, 2002). Es decir, podemos llegar a una sistematización 
de ideas para constituir la objetividad de nuestro estudio, basados en el fenómeno social que presentamos, dentro de un marco en el que la realidad es muy particular.

Por otra parte, en el marco metódico el diseño aplicado es transeccional o transversal; recolectando datos en un momento y espacio único, con el propósito de describir: variables, interrelación, incidencia, etc. (Albert Gómez, 2007). Asimismo, este diseño permite describir los hechos por medio de inferencias entre la(s) variable(s) que vamos a usar, desde el punto de vista más óptimo que el investigador considere, ya sea entre correlación de variables, contextos o simplemente analizar una variable.

\subsection{Población y muestra}

La muestra de una población es requerida para limitar un estudio; la delimitación permite inferir resultados y establecer parámetros. En nuestra investigación el tipo de muestra es probabilística, ya que se requirió precisar el tamaño de la población. Herández y cols. (2014) aseguran que la muestra depende de quién(nes) investiga(n), partiendo del interés investigativo para centrar a qué o quién(nes) como muestra. Asimismo, la muestra depende no solo de los objetivos de la investigación, sino también de su diseño, lo que, posteriormente, permitirá su análisis, descripción e interpretación.

En este sentido, la muestra total de participantes en nuestro estudio es de $\mathrm{N}=528$. En cuanto a docentes masculinos del área urbana es $\mathrm{n}=17$, y de docentes femeninos es $n=476$. Por otra parte, en el área rural los docentes masculinos son $n=3$, y docentes femeninos $n=32$.

Tabla 1: Población y muestra del estudio presentado

\begin{tabular}{lccc}
\hline & $\begin{array}{c}\text { Población } \\
(\mathrm{N})\end{array}$ & $\begin{array}{c}\text { Muestra } \\
(\mathrm{n})\end{array}$ & Porcentaje \\
\hline Docentes femeninos (área urbana) & 493 & 476 & $96,55 \%$ \\
Docentes masculinos (área urbana) & & 17 & $3,45 \%$ \\
Docentes femeninos (área rural) & 35 & 32 & $91,43 \%$ \\
Docentes masculinos (área rural) & & 3 & $8,57 \%$ \\
\hline Total de docentes & 528 & & \\
\hline
\end{tabular}

\subsection{Instrumentos}

El Archivo Maestro de Instituciones Educativas (AMIE), recolecta datos de instituciones públicas y privadas es decir datos de: estudiantes, docentes, infraestructura, modalidad, sector, entre otros; a nivel nacional y de territorio (Ministerio de Educación, 2020), tanto al inicio como al final del año escolar. Este registro funciona desde el período 2007-2008 reemplazando al Sistema Nacional de Estadísticas Educativas (SINEC). La información se encuentra actualizada desde el periodo 2009-2010 al 20182019.

\subsection{Procedimiento de recogida y análisis de datos}

A través de la revisión del código AMIE, se logró obtener los datos de las instituciones que atienden a niños de Educación Inicial de todo el Ecuador. Sin embargo, la presente investigación se centró en la provincia del Azuay, en 100 Centros de Educación Inicial con un número de 528 docentes que atienden a la primera infancia -entre docentes masculinos y femeninos-.

Obteniendo los resultados que se analizarán en el siguiente apartado a través de la exposición de los contenidos realizados en el programa de Excel, el cual permitió cuantificar el número de CEI de zonas urbanas y rurales, el tipo de atención, parroquia o cantón, tipo de servicio: privado o público y, sobre todo, permitió dar relevancia al estudio permitiendo analizar la cantidad de docentes de género masculino que trabajan con niños entre 0 a 5 años de edad en la provincia del Azuay - Ecuador.

\section{Resultados}

La tabla número 2 corresponde a las 15 parroquias urbanas de la provincia del Azuay, las cuales sumadas poseen 91 Centros de Educación Inicial, tanto del sector público como privado, de carácter escolarizado y de educación regular; cuya modalidad es netamente presencial y bifurca entre jornada matutina y vespertina. Estos CEI permiten evidenciar la minoría en cuanto a la presencia de hombres laborando en Educación Inicial; existen una cantidad de 17 varones y 476 mujeres trabajando en estos centros. Es decir, apenas el 3,45\% de docentes de la zona urbana corresponde a varones, evidenciando así la preponderancia en número de la mujer en el campo de la Educación Inicial, con un porcentaje de $96,55 \%$, ya sea por motivos sociales, culturales u opresores.

Sin embargo, las nuevas masculinidades están siendo visibilizadas en un rango minúsculo pero presente, rompiendo estereotipos y luchando contra un sistema lleno de creencias e imaginarios, lo cual permite afirmar la necesidad del presente estudio.

Tabla 2: Docentes de instituciones educativas urbanas en la provincia del Azuay - Ecuador

\begin{tabular}{|c|c|c|c|c|c|c|c|c|}
\hline \multicolumn{9}{|c|}{ Parroquias Urbanas del Azuay } \\
\hline Instituciones & Cod_Provincia & Escolarización & $\begin{array}{c}\text { Tipo } \\
\text { Educación }\end{array}$ & Zona INEC & Modalidad & $\begin{array}{l}\text { Docentes } \\
\text { Femenino }\end{array}$ & $\begin{array}{c}\text { Docentes } \\
\text { Masculino }\end{array}$ & $\begin{array}{c}\text { Total } \\
\text { Docentes }\end{array}$ \\
\hline
\end{tabular}


También se ha realizado un estudio en Ecuador, con estudiantes varones de la carrera de Educación Inicial de la Universidad Nacional de Educación, en el que el $100 \%$ de los estudiantes y el $75 \%$ de docentes a quienes se les realizó una entrevista, consideran que la carrera de Educación Inicial se encuentra vinculada al cuidado y la pedagogía (Morocho, 2018) puesto que se relaciona con el proceso de aprendizaje, en donde el niño menor a cinco años aprende de manera cultural y que su parte cognitiva necesita de estos procesos. Además, Morocho (2018) afirma que la concepción que las personas en formación del profesorado poseen sobre el desarrollo infantil integral debe partir de una base conceptual, estructural, de formas, abstracciones, ideologías, y por sobre todo de la comprensión de cosas nuevas, para evitar miradas androcéntricas, discriminación y violencias social basadas en estereotipos.

Boscán, 2008 considera que se debe rescatar los aspectos positivos de la masculinidad, especialmente aspectos o características que permitan al hombre mantener una buena autoestima y confianza, además, durante las últimas dos décadas varios hombres, se han convertido en personas más tranquilas y pacientes. El autor aboga por que la actitud pacífica en el hombre no afecte o disminuya su energía masculina, al contrario, lo hace más humanista, tolerante, comprensivo, capaz de entender que la educación debe ser la herramienta para erradicar imaginarios sociales en los que la desigualdad y discriminación priman. El cambio y la concientización se da al derribar barreras o imaginarios que produce la sociedad, y es la responsabilidad del ser humano cambiar ideas que permitan al individuo trabajar en una profesión que lo complemente y no sea criticado o juzgado por ejercerla.

Murcia, 2012 apoya el currículo de educación colombiana, el cual hace énfasis en la formación de competencias sociales como "el camino para la paz". Su idea se encuentra fundamentada en las recomendaciones de la UnescoCepal, en las que se establece una relación entre educaciónconocimiento, consideradas como eje de la transformación productiva y equidad; poseen como base las perspectivas de aprendizaje social-cognitivo. Las conductas sociales las adquirimos con base en el otro, estas conductas pueden ser copiadas o pueden llegar a inhibirse en el sujeto y en el caso de la escuela, en el niño.

La Educación Inicial es una de las etapas escolares más importantes del ser humano, puesto que, por sus diversos procesos de plasticidad cerebral, el niño absorbe no sólo la parte cognitiva sino también actitudinal observada en su figura élite, el docente. Además, a esta edad se genera la empatía humana y es ahí donde se debe deconstruir imaginarios sociales que, a futuro, causan desigualdades, violencia o discriminación en los roles -impuestos por estereotipos que obligan a las personas a cumplir determinadas funciones, acciones y comportamientos- que se cumple en la sociedad.

Otra de las aristas que rigen en el campo de la educación, es el factor económico, ya que la sociedad aún considera que el hombre debe ganar un salario mayor que la mujer, es ahí donde esta variable, influye en las decisiones del varón al momento de escoger su profesión, la brecha que existe entre salarios, es incalculable. Buchelli y Sanroman, 2004 describen la existencia de un techo de cristal para las mujeres de Uruguay, demuestran que los salarios femeninos se distancian de los masculinos especialmente en el campo privado. Es importante buscar la presencia de un techo de cristal para analizar la diferencia salarial entre hombres y mujeres de distintas profesiones, lo que probablemente tenga razones estereotipadas socialmente.

Los imaginarios sociales pueden incluir o excluir a una persona en los diferentes aspectos y espacios de la vida, además de causar posibles mejoras en su vida, o al contrario, pueden causar daños o perjuicios en un individuo, ya que en la mayoría de las ocasiones, los estereotipos concebidos limitan el actuar de una persona, cortan los sueños, ideales o metas que se proponen, puesto que la hegemonía establecida por la sociedad del hombre ante la mujer, conllevan a una pugna de poder que lastimosamente daña tanto a la persona misma como a un par.

Pintos, 2004 afirma que las sociedades policontexturales, al no poseer una sola identidad, y un solo lenguaje, obliga a una sociedad a utilizar determinados conceptos y crea una manera paradójica de formulaciones sociales, trivializan ideas y conceptos que confunden a la persona para actuar en determinadas situaciones, incluyendo la moral pública y a las buenas acciones que se encuentran dentro de un margen de convivencia y, aceptadas en una sociedad, perjudican a la persona en su toma de decisiones, puesto que no respeta la autonomía del individuo y su libre albedrío.

La sociedad, por lo tanto, es un juez que critica y discrimina acciones, socialmente no aceptadas, donde el hombre cumple acciones descritas por una sociedad y por un poder o una fuerza, etiquetando y catalogando en una serie de acciones que no le permiten asumir el rol que desea, la sociedad afecta desde la infancia al etiquetar por colores, juegos, vestimenta a la persona y delimita sus ideales y preferencias, al considerar superior al niño de la niña e irrespetando la igualdad de trato desde edades tempranas.

Entendemos que la vida es una constante búsqueda de la verdad, qué está bien y qué no, qué es real y qué no, hay muchas preguntas ¿cómo responderlas? Las investigaciones de diversas áreas permiten tener un panorama de la sociedad en el que se refleja desigualdades, avances, acciones de todo tipo; hay hechos positivos, al igual que negativos, pero estas dos etiquetas: bien o mal ¿desde cuándo dominan nuestras vidas? Acaso son producto de nuestra imaginación, y si lo son, quizá la parte que responde al significado otorgado a lo que es bueno es nuestra humanidad, la fraternidad que tenemos unos con otros, y lo que responde a lo que está mal probablemente fue la causa de guerras.

Toda acción, sin etiquetas, tendrá el significado que una persona decida darle, después de todo, vivimos en 
un mundo instituido de significados, ninguno absoluto, siempre habrá cambios. Nuestra imaginación ha sido la herramienta principal para crear realidades, más allá de entender una lógica, podemos crear más más realidades, tan infinitas como nuestra imaginación lo permita.

El centro, es que lo que imaginamos lo compartimos, cuántos grandes inventos no fueron materializados después de imaginarlos, y al ser compartidos, son elementos sociales. El rol social queda en que, incluso la imaginación es parte fundamental en el camino hacia el descubrimiento de la verdad. En este sentido, los imaginarios sociales son la unión de diversos pensamientos, ideas, que fueron consolidadas hasta fusionarse y materializarse, en una palabra, o en una etiqueta; el rol de la educación es que cada palabra debe ser manejada como un bisturí en una operación, con extremo cuidado.

El imaginario que posea la parte docente es que el reflejará hacia sus discentes, ventajosamente la familia educativa es amplia y ha estado reinventándose por mucho tiempo, por ende, los imaginarios sociales deben seguir el mismo proceso, cambiar, actualizarse, y este cambio, sin duda alguna, debe ser intervenido por buenas y cambiantes practicas docentes al igual que sociales. El uso de significados debe darse a palabras y/o acciones, pero las etiquetas crean desigualdad, marcan a minorías, tenemos que imaginar cómo se sienten estas minorías, pues para que haya realidad, debe haber imaginación.

Es decir, ser empáticos y aceptar la diversidad de personas, acciones, hechos, roles, de todo, en todo su esplendor y al fin, reinventarnos, ser mejores, imaginemos cosas mejores, aquel mundo utópico en el que no existan minorías, grupos, al contrario, gente siendo y haciendo lo que le gusta.

\section{Referencias Bibliográficas}

Albert Gómez, M. (2007). La investigación educativa. Claves teóricas. Madrid: Mc Graw Hill.

Ayala, M. (2007). Masculinidades en el campo. Ra Ximhai, 3(3), 739-761.

Boscán, A. (2008). Las nuevas masculinidades positivas. Revista Internacional de Filosofía Iberoamericana y Teoría Social(41), 93-106.

Buchelli, M., y Sanroman, G. (2004). Salarios femeninos en el Uruguay ¿Existe un techo de cristal? Uruguay: Universidad de la República.

Canto, E. D., y Silva, A. (2012). Metodología cuantitativa: abordaje desde la complementariedad en Ciencas Sociales. Revista de Ciencias Sociales, 3(141), 2534.

Cappi, L., y Salle, M. (2018). Docencia Masculina en la educacióninfantil: Discurso de negación de lacomunidad escolar. Subjetividad y Procesos Cognitivos, 2(22).

Cappi, L., y Salles, M. (2014). Profesoras y docencia de estudiantes del sexo masculino de práctica profesio- nal en una guardería: desafíos en las relaciones entre géneros. Subjetividady Procesos cognitivos, 71-97.

Cornachione, M. (2016). Psicología del Desarrollo: aspectos biológicos, psicológicos y sociales . Córdoba: Editorial Brujas.

Cuadernos UCAB. (1997). Lev Vygotsky: sus aportes para el siglo XXI. Caracas: Universidad Catolica Andres Bello.

Gerrig, R., y Zimbardo, P. (2005). Psicología y vida. México: Pearson Educación.

Herández, R., Fernández, C., y Batista, P. (2014). Metodología de la investigación. México: McGraw-Hill.

Kerlinger, F., y Howard, L. (2002). Investigación del comportamiento. Métodos de investifación en ciencias sociales. México: McGraw-Hill.

Lenzi, A., Borzi, S., y Tau, R. (2010). El concepto de desarrollo en psicología: entre la evolución y la emergencia. Fundamentos en Humanidades, XI(22), $139-163$.

Lizana, V. (2007). No Title. Chile: Universidad de Chile.

Ministerio de Educación. (2014). Currículo Educación Inicial. Quito, Ecuador.

Murcia, N. (2012). La escuela como imaginario social. Apuntes para una escuela dinámica. Magistro, 6(12), 53-70.

Navarro, I., y Pérez, N. (2012). Psicología del desarrollo humano: del nacimiento a la vejez. San Vicente del Raspeig: Editorial Club Universitario.

Nuñez, G. (2007). Masculinidad e intimidad: identidad, sexualidad y sida. México: Miguel Ángel Porrúa.

Núñez, G. (2016). Los estudios de género de los hombres y las masculinidades: ¿qué son y qué estudian? Culturales, 4(1), 9-31.

Organización de Naciones Unidas. (2018). Objetivos de Desarrollo Sostenible. Descargado de https:// www . un.org/sustainabledevelopment / es/2018/08/sabes-cuales-son -los-17-objetivos-de-desarrollo - sostenible/

Pintos, J. (2004). Inclusión-exclusión. Los imaginarios sociales de un proceso de construcción social. SeMata, Ciencias sociais e humanidades, 16, 17-52.

Rego, A., y Pereira, H. (2004). Cómo los comportamientos de ciudadanía docente explican la eficacia pedagógica y la motivación de los estudiantes. Revista Interamericana de Psicología, 38(2), 201-216.

Rice, P., y Ortiz, M. (1997). Desarrollo humano: estudio del ciclo vital. México: Pearson Educación.

Szurmuk, M., y McKee, R. (2009). Diccionario de estudios culturales latinoamericanos. México: Siglo XXI.

Taylor, C. (2004). Modern Social Imaginaries . North Caroline: Duke University Press.

UNESCO. (2019). Las metas educativas. Descargado de https://es.unesco.org/node/266395

UNICEF. (2017). La primera infancia importa para cada niño. UNICEF, 1-77. 
Valhondo, A. M. (1995). Psicología del desarrollo y de la educación en la edad escolar. Oviedo: Universidad de Oviedo.

Vásquez, H., y Méndez, P. (2016). Imaginarios sociales en torno al comportamiento ciudadano docente: una mirada desde la formación inicial docente. Actualidades Investigativas en Educación, 16(1), 1-32.

Vendrell, R., Dalmau, M., Gallego, S., y Baqués, M. (2015). Los varones, profesionales en la educación infantil. Implicaciones en el equipo pedagógico y en las familias. Revista de Investigación Educativa, 195-210.

Recibido: 12 de agosto de 2020

Aceptado: 15 de noviembre de 2020

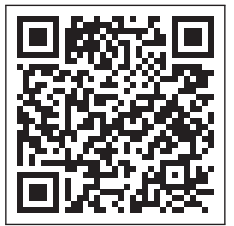


